\title{
Reliability and validity study of measurements on digital photography to evaluate shoulder balance in idiopathic scoliosis
}

\author{
Antonia Matamalas ${ }^{1 *}$, Juan Bagó ${ }^{1}$, Elisabetta D'Agata ${ }^{2}$ and Ferran Pellisé ${ }^{1}$
}

\begin{abstract}
Objective: To determine the validity of digital photography as an evaluation method for shoulder balance (ShB) in patients with idiopathic scoliosis.

Material and methods: A total of 80 patients were included (mean age 20.3 years; $85 \%$ women). We obtained a full $x$-ray of the vertebral column and front and back clinical photography for all patients. For antero-posterior $x$-rays we measured the proximal thoracic curve angles (CPT). To evaluate radiological shoulder balance we calculated the clavicle-rib intersection angle (CRIA) and T1-tilt. For clinical photography we measured shoulder height angle (SHA), axilla height angle (AHA) and the left right trapezium angle (LRTA). We analyzed the reliability of the different photographic measurements and the correlation between these and the radiological parameters.

Results: The mean magnitude of PTC, CRIA and T1-tilt were $19^{\circ},-0.6^{\circ}$ and $1.4^{\circ}$ respectively. Mean SHA from the front was $-1.7^{\circ}$. All photographic measurements revealed an excellent-near perfect intra and inter-observer reliability in both photographic projections. No correlation was found between the ShB and the magnitude of the PTC.

A statistically significant correlation was found between clinical balance of the shoulders and radiological balance ( $r$ between 0.37 and 0.51).
\end{abstract}

Conclusions: Digital clinical photography appears to be a reliable method for objective clinical measurement of ShB. The correlation between clinical and radiological balance is statistically significant although moderate/weak.

Keywords: Cosmetic, Idiopathic scoliosis, Photography, Shoulders balance

\section{Introduction}

Cosmetic disorder is one of the main reasons to treat Idiopathic Scoliosis patients (SOSORT Consensus) [1]. Shoulder balance $(\mathrm{ShB})$ has been considered a characteristic of the deformity in idiopathic scoliosis [2-4]. According to Raso, this represents $75 \%$ of the perceived deformity of the trunk, together with asymmetry of the scapulae and shoulder girdle [2].

To be able to evaluate this balance correctly, reliable tools are necessary. Different evaluation methods ranging from radiological, clinical and topographical have been proposed over the years. Hong et al. [5] recently evaluated the reliability and validity of the different radiological

\footnotetext{
*Correspondence: amatamalasadrover@gmail.com

'Department of Orthopaedic Surgery, Hospital Vall d'Hebron, P Vall d'Hebron, 119, 08035 Barcelona, Spain

Full list of author information is available at the end of the article
}

methods to evaluate ShB and concluded that, in general, all outcomes have reliable intra and inter-observer reliability. Nonetheless, radiological balance does not appear to optimally correspond with clinical balance, which suggests that clinical parameters should be a complement to radiological outcomes [6].

Different methods have been proposed to assess shoulder imbalance [2-4]. Zaina et al., have developed a tool for routine clinical use (TRACE), consisting of photographs depicting different severities of four aspects of trunk deformity; shoulder, scapulae, hemithorax and waist. This instrument relies on subjective impression of the observer [7]. Surface topometry such as Moiré-Fringe or 3D scan (Vitrus) [8] methods have been used. Nonetheless, these systems require expensive equipment and a trained operator, which means that their usefulness in clinical practice 
remains a moot point. Conversely, clinical photography corrects some of these defects: the equipment is cheap, simple to handle and quick to obtain. These reasons lead to supposing that it may be of considerable interest in daily practice.

Two indices have traditionally been used to study the shoulders in clinical photography; the shoulder height difference (in $\mathrm{cm}$ or by means of the angular measurement) and the axillary fold height difference [9-11]. Other indices to evaluate $\mathrm{ShB}$ have recently been proposed shoulder height difference at the level of the upper border of the trapezium muscle $[6,9,11,12]$ and the area of the trapezium muscles [12]. The problem with these latter indices lies in the fact that each author defines them differently. Furthermore, data on the reliability of these outcomes are incomplete because no inter-observer reliability data for any of them are available, especially for front-view photography. We think it is necessary to ascertain the validity of the measurements taken from the front because this view corresponds to the patient's view when they look in a mirror.

The aims of our research are twofold: a. to determine the test-retest reliability of various clinical measurements taken with digital photography and to compare the data between front and rear shots; b. to determine the validity of these photographic measurements by analyzing their relationship with the radiological measurements of ShB.

\section{Materials and methods}

This is a transversal study approved by the clinical research ethics committee of Hospital Vall d'Hebrón. The inclusion criteria for this study were patients with idiopathic scoliosis with a largest Cobb angle (MLC) greater than $25^{\circ} \mathrm{Cobb}$ in the coronal plane, aged between 12 and 40 who agree to take part in the study. Patients were recruited consecutively; only patients who had not received surgery were included. At the time the picture was taken, no patient was on an active treatment with brace. The sample was stratified according to MLC into two groups: Group $<45^{\circ}$ and Group $\geq 45^{\circ}$. This cut-off value of $45^{\circ}$ was chosen because at this magnitude, surgical treatment can be recommended. For each patient there was a posteroanterior $\mathrm{x}$-ray of the full trunk in standing position performed the week before taking part in the study.

\section{Radiological measurements}

The following was recorded on postero-anterior x-ray: the magnitude of the proximal thoracic (PTC), main thoracic (MTC) and thoraco-lumbar/lumbar (TLLC) curves. Furthermore, the tilt with respect to the horizontal of T1 (T1-tilt) (Figure 1), the lower end vertebra of the PTC (PTC_LEV), the lower end vertebra of the MTC (MTC_ LLV) and lower end vertebra of the TLLC (TLLC_LLV) (Figure 2), was measured. Radiological ShB was calculated by means of the Clavicle-Rib Intersection Angle (CRIA). CRIA is defined as the angle formed by the horizontal and

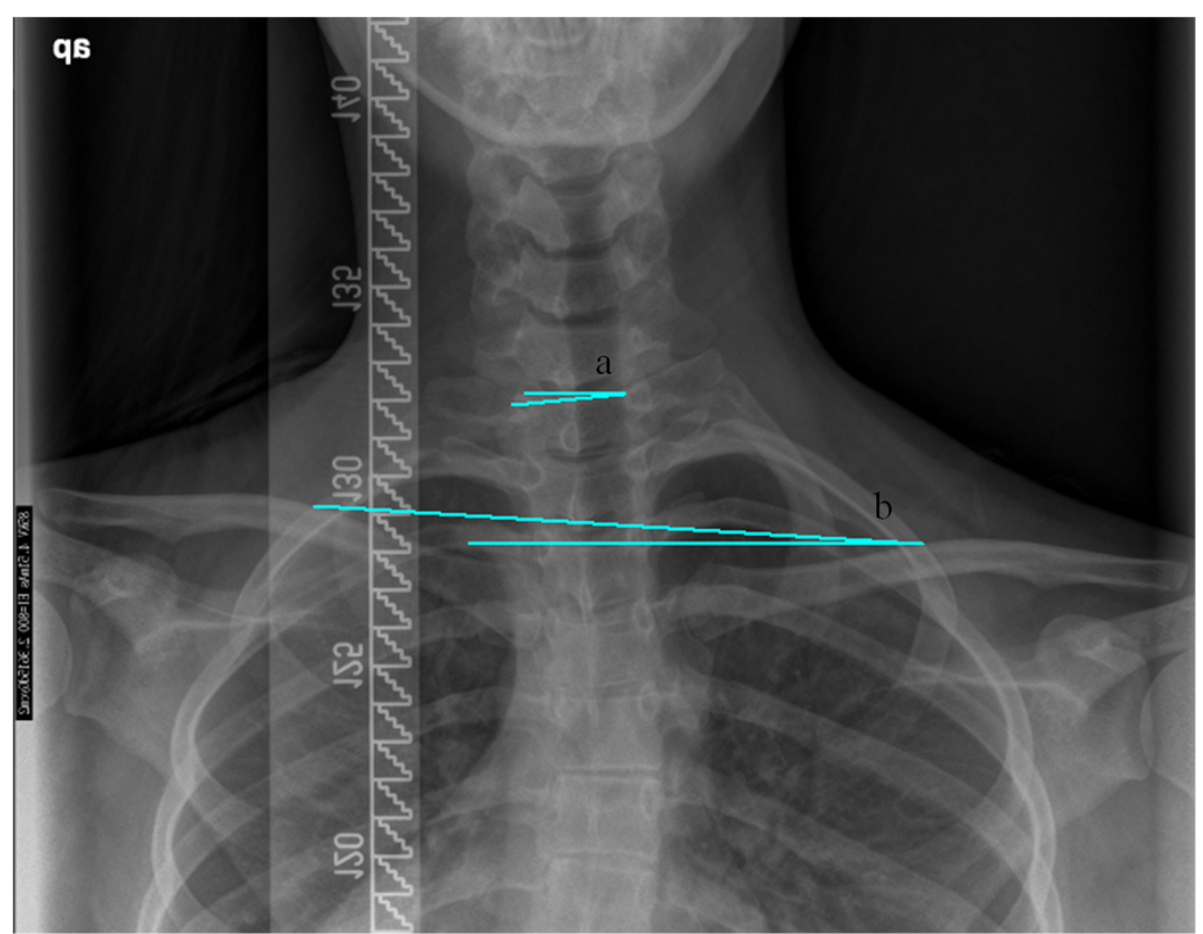

Figure 1 Radiological measurements of shoulder balance. (a) T1-tilt (b) Clavicle-rib intersection angle (CRIA). 


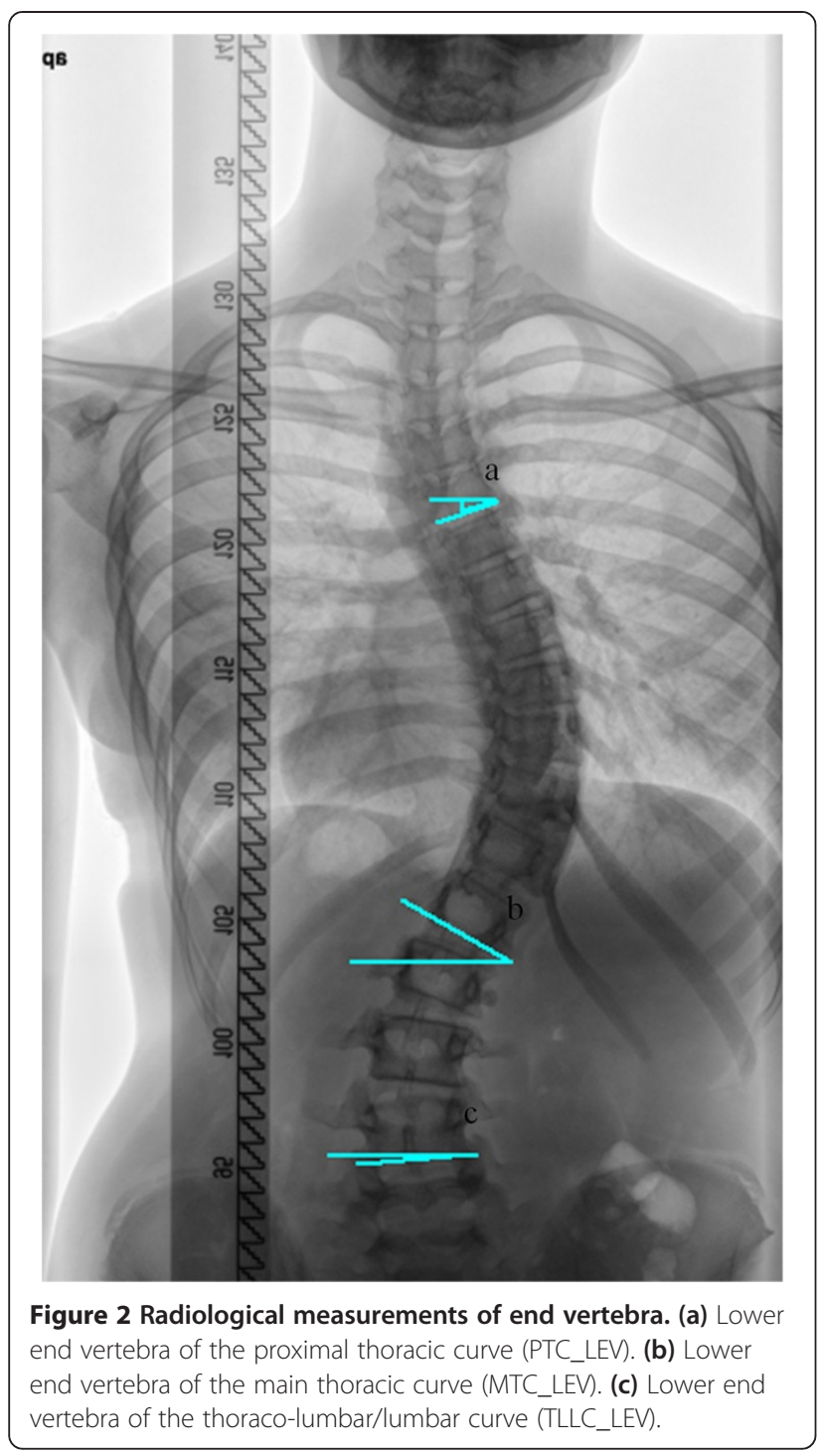

a line that joins the intersection points of the clavicles with the rib-cage (Figure 1).

\section{Photographic measurements}

Each patient underwent clinical photographs on the same day of the visit by just one trained examiner (EA) who undertook the entire process.

To acquire the photographs a digital Nikon D5100 (Nikon Corporation, Tokyo, Japan) camera, mounted on a tripod at 110 centimeters in height and with a distance of 130 centimeters, was used for both photographs. The patients' position was standardized on a previously marked cross on the floor. Patients were told to adopt a relaxed standing position when the photographs were taken. All of them were photographed with an anterior (front) and posterior (back) view.
For each photograph three photographic indices were calculated:

Left/right trapezium angle (LRTA): The trapezium angle is defined as the angle between the line following the external border of the trapezium muscle and the horizontal. The left/right ratio of this angle was used for statistical analysis (Figures 3 and 4).

Shoulder height angle (SHA): Angle formed between the line that joins the upper border of both acromion processes and the horizontal (Figures 3 and 4).

Axilla height angle (AHA): Angle formed between the line that joins the upper border of both external axillary folds and the horizontal (Figures 3 and 4).

The SurgimapSpine ${ }^{\circ}$ (Nemaris Inc, New York, United States) software was used for both the $\mathrm{x}$-ray and clinical photography measurements. Both the radiological and photographic measurements were assigned a positive or negative value according to the tilt direction. The right-hand thumb rule was used for this: looking at the individual (or $\mathrm{x}$-rays) from the back, a clockwise and anti-clockwise tilt was considered positive and negative, respectively. For example, the tilt of the end vertebrae towards the right was assigned a positive value; elevation of the left shoulder was assigned a positive value; curves with convexity to the left and right were assigned a positive and negative value, respectively.

\section{Statistical analysis}

Descriptive statistics (mean and range) were used to report patients and radiological and photographic measurements.

To determine the reliability of the photographic indices an intra and inter-observer reliability analysis using the intraclass correlation coefficient (ICC) with absolute agreement and a 95\% confidence interval, was performed. To analyze reliability photographs of the first 60 patients (30 cases with MLC $<45^{\circ}$ and 30 cases with MLC $>45^{\circ}$ ) were used. Each photograph was measured by three evaluators (AM, JB, EA) on two separate occasions, one week apart. To calculate intra-observer reliability all three observers' measurements were analyzed (in total 180 measurements). The first and second measurements of all observers were jointly compared. To calculate inter-observer reliability the first measurement made by each observer was used. The intra and interobserver intra-class correlation coefficient was obtained for each measurement. According to Landis \& Koch [13] the following scale was used to interpret the ICC: $<0.20$ minimal or inexistent relationship; $0.21-0.40$ poor; 0.41 - 0.60 moderate; $0.61-0.80$ excellent and $>0.81$ near perfect.

To study convergent validity, Pearson correlation coefficients between the photographic and radiological measurements were calculated. 


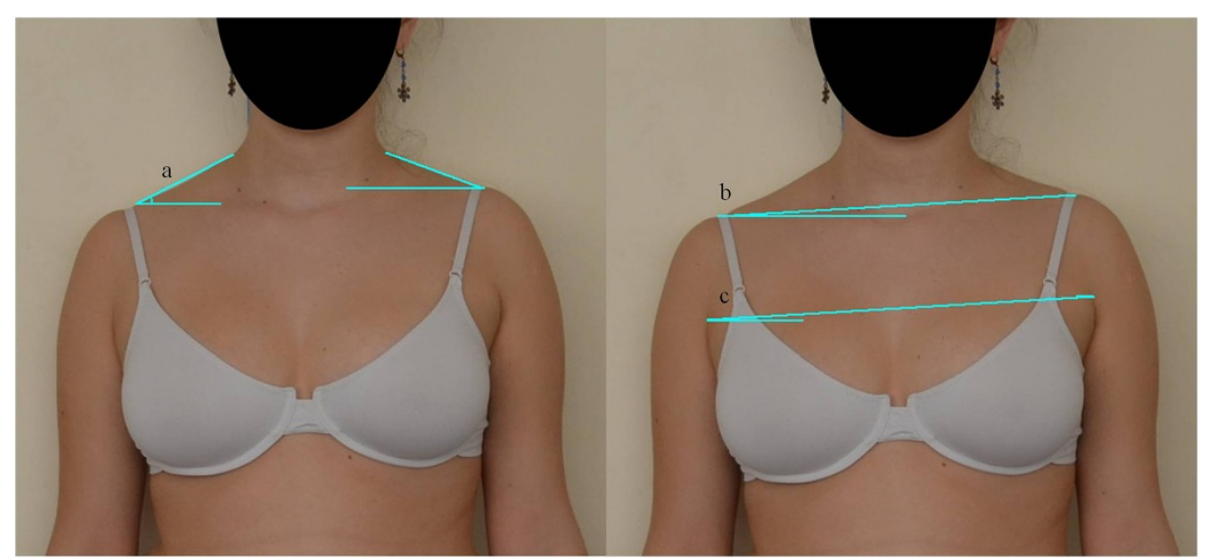

Figure 3 Photographic measures in front view. (a) Right and left trapezium angle. (b) Shoulder height angle. (c) Axilla height angle.

To test the degree of equivalence of the back and front SHA and the degree of equivalence between the front SHA and AHA, the concordance correlation coefficient was determined $[14,15]$. Similar to the Pearson coefficient, the CCC varies between -1 and +1 . The criteria recommended by McBride were used to determine the degree of concordance: >0.99 Near perfect; 0.95-0.99 Substantial; 0.90-0.95 Moderate and $<0.90$ Poor [16].

Data were processed using a SPSS 17.0 program. $P<0.05$ was considered statistically significant.

\section{Results}

\section{Sample descriptions}

A total of 80 consecutive patients were included. Mean patient age (typical deviation) was 20.3 years $( \pm 8.6)$ and $85 \%$ of patients were women. A total of $68.8 \%$ of patients had attained skeletal maturity (Risser 4 and 5) at the time of inclusion into the study and $16.3 \%$ were immature patients (Risser 1 and 2). The distribution of frequencies of the different kinds of curve according to the Lenke classification [17] was: type 1 (27.5\%), type 2 (5\%), type 3 (26.3\%), type $4(2.5 \%)$, type $5(32.5 \%)$ and type $6(6.3 \%)$. Table 1 shows the means and range of different radiological and photographic measurements.

\section{Reliability and standard error of measurement}

Table 2 represents intra and inter-observer reliability values and the standard error of measurement for the different photographic measurements. As can be seen in the table, the intra-observer ICC values for SHA and AHA both front and back were $>0.80$ indicating a near perfect correlation; for LRTA the intra-observer ICC were excellent ( 0.79 and 0.78 respectively). The inter-observer ICC were slightly less although within the near perfect correlation range $(>0.80)$ except for the front LRTA which was excellent $(0.65)$.

\section{Concordance}

The SHA and AHA angles in the photograph taken frontally presented poor concordance (CCC 0.66; 95\%

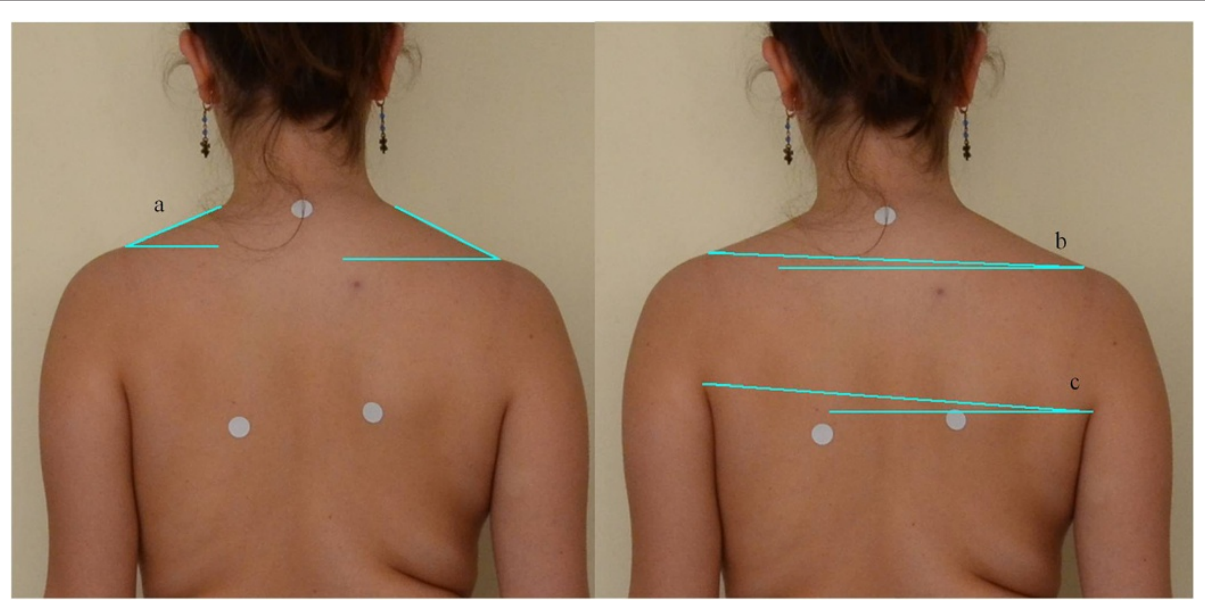

Figure 4 Photographic measures in back view. (a) Right and left trapezium angle. (b) Shoulder height angle. (c) Axilla height angle. 
Table 1 Descriptions of the radiological and photographic outcomes

\begin{tabular}{|c|c|c|c|}
\hline \multirow[t]{2}{*}{ Variables } & \multirow[t]{2}{*}{ Mean $\left({ }^{\circ}\right)$} & \multicolumn{2}{|l|}{ Range } \\
\hline & & Minimum & Maximum \\
\hline \multicolumn{4}{|c|}{ Radiological } \\
\hline PTC & 18.9 & -15.9 & 46.3 \\
\hline PTC_LEV & -17.4 & -42 & 19.2 \\
\hline MTC & -33.5 & -78.0 & 53.2 \\
\hline MTC_LEV & 16.5 & -35 & 42.1 \\
\hline TLLC & 24.1 & -55.6 & 60.9 \\
\hline TLLC_LEV & -7.7 & -33.7 & 36.9 \\
\hline T1 tilt & 1.4 & -16.9 & 29.2 \\
\hline CRIA & -0.6 & -12.1 & 7.3 \\
\hline \multicolumn{4}{|c|}{ Photographic } \\
\hline LRTA back & 1.1 & 0.7 & 2.0 \\
\hline SHA back & -0.7 & -8.4 & 6.2 \\
\hline AHA back & -1.8 & -8.2 & 7.9 \\
\hline LRTA front & 1.2 & 0.6 & 3.4 \\
\hline SHA front & -1.7 & -11.1 & 5.5 \\
\hline AHA front & -2.3 & -11.9 & 6.4 \\
\hline
\end{tabular}

PTC (Cobb proximal thoracic curve); PTC_LEV (Lower end vertebra of the proximal thoracic curve); MTC (Cobb main thoracic curve); MTC_LEV (Lower end vertebra of the major thoracic curve); TLLC (Cobb thoraco-lumbar/lumbar curve); TLLC_LEV (Lower end vertebra of the thoraco-lumbar/lumbar curve); T1 tilt (T1 inclination angle); CRIA (clavicle-rib intersection angle); LRTA (Left/right trapezium angle ratio); SHA (Shoulder height angle); AHA (Axilla height angle).

$\mathrm{CI}=0.53-0.76)$. The same occurs with the SHA angles for front and back whose concordance was also poor (CCC 0.49; 95\% CI = 0.32-0.64).

\section{Correlations between photographic and radiological measurements}

Table 3 lists the correlation coefficients between the photographic and radiological measurements. The correlation of photographic measurements with radiological

Table 2 Intra and inter-observer reliability and standard error of measurement (SEM) of the photographic measurements

\begin{tabular}{llll}
\hline Variable & $\begin{array}{l}\text { Intra-observer } \\
\text { reliability (ICC) }\end{array}$ & $\begin{array}{l}\text { Inter-observer } \\
\text { reliability (ICC) }\end{array}$ & $\begin{array}{l}\text { Standard error of } \\
\text { measurement (SEM) }\end{array}$ \\
\hline Back & & & \\
\hline LRTA & 0.79 & 0.80 & 0.14 \\
SHA & 0.88 & 0.80 & 0.99 \\
AHA & 0.93 & 0.88 & 0.83 \\
\hline Front & & & \\
\hline LRTA & 0.78 & 0.65 & 0.24 \\
SHA & 0.91 & 0.89 & 1.06 \\
AHA & 0.91 & 0.85 & 1.18 \\
\hline
\end{tabular}

LRTA (Left/right trapezium angle ratio); SHA (Shoulder height angle); AHA (Axilla height angle). parameters was poor to moderate. Only PTC correlated poorly $(r=-0.26)$ with AHA for the back shot; there was no correlation with any other photographic parameter. For MTC we observed a moderate correlation $(r=0.46)$ with AHA posterior view; there were poor correlations with the frontal view clinical parameters ( $\mathrm{r}$ ranging from -0.27 to 0.27 . TLLC was moderately to poorly correlated with all photographic parameters both for front and back view ( $\mathrm{r}$ ranging from -0.45 to 0.35 ). The end vertebrae of the different curves revealed similar correlations to those of the overall magnitude of the curve.

The radiological measurement of ShB (CRIA) showed moderate correlation with the photographic measurements, especially the frontal photograph (LRTA $r=-0.45$; SHA $r=0.48$ y AHA $r=0.51$ ). A statistically significant correlation was found between the photographic measurements and T1-Tilt, especially for the SHA in the frontal view $(r=0.51)$.

\section{Discussion}

Shoulder balance is considered characteristic of idiopathic scoliosis. Use of clinical photography to measure ShB has not been fully analyzed. Clinical photography offers a series of practical advantages: it is cheap, simple to handle and images are almost immediately available. The aims of our research were to determine the reliability of various measurements taken using digital photography and to evaluate their relationship with radiological parameters in a non-selected population of patients with idiopathic scoliosis.

\section{Selection of measurements}

An initial step to design the research was to decide which measurement to include in the study. It was decided to select angular measurements to avoid problems from the calibration necessary when using linear measurements. In the case of asymmetry of the trapezium muscles, it was preferred to use angular measurements instead of surface areas as we believe that the latter is more complex and not very useful in daily clinical practice. We also ruled out using skin markers as other authors had done previously. We believe that this methodology lengthens examination time and introduces a new source of bias. We therefore preferred to define, a priori, the anatomic points to be used as a reference for the measurements.

After these prior considerations, it was decided to record three parameters:

1. Shoulder height angle (SHA): formed between the line that joins the upper border of both acromion processes and the horizontal. This is the parameter which should, a priori, collate shoulder imbalance better. This parameter has been used previously by other researchers both for front $[9,12]$ and back 
Table 3 Correlation between the clinical outcomes of imbalance of the shoulders and the radiology

\begin{tabular}{|c|c|c|c|c|c|c|c|c|c|c|c|c|}
\hline \multicolumn{13}{|c|}{ Correlations } \\
\hline & \multicolumn{6}{|l|}{ Back } & \multicolumn{6}{|l|}{ Front } \\
\hline & \multicolumn{2}{|l|}{ LRTA } & \multicolumn{2}{|l|}{ SHA } & \multicolumn{2}{|l|}{ AHA } & \multicolumn{2}{|l|}{ LRTA } & \multicolumn{2}{|l|}{ SHA } & \multicolumn{2}{|l|}{ AHA } \\
\hline & $r$ & $p$ & $r$ & $p$ & $r$ & $p$ & $r$ & $p$ & $r$ & $p$ & $r$ & $p$ \\
\hline CRIA & -0.35 & 0.00 & 0.39 & 0.00 & 0.35 & 0.00 & -0.45 & 0.00 & 0.48 & 0.00 & 0.51 & 0.00 \\
\hline T1 & -0.35 & 0.00 & 0.37 & 0.00 & 0.31 & 0.005 & -0.35 & 0.00 & 0.51 & 0.00 & 0.44 & 0.00 \\
\hline PTC & -0.07 & n.s. & 0.03 & n.s. & -0.26 & 0.02 & -0.07 & n.s. & 0.04 & n.s. & -0.03 & 0.02 \\
\hline MTC & -0.16 & n.s. & 0.19 & n.s. & 0.46 & 0.00 & -0.27 & 0.02 & 0.24 & 0.03 & 0.27 & 0.01 \\
\hline TLLC & 0.35 & 0.002 & -0.33 & 0.003 & -0.45 & 0.00 & 0.23 & 0.04 & -0.26 & 0.02 & -0.30 & 0.007 \\
\hline PTC_LEV & -0.11 & n.s. & 0.17 & n.s. & 0.45 & 0.00 & -0.27 & 0.15 & 0.25 & 0.02 & 0.29 & 0.008 \\
\hline MTC_LEV & 0.19 & n.s. & -0.19 & n.s. & -0.42 & 0.00 & 0.24 & 0.29 & -0.21 & n.s. & -0.24 & 0.03 \\
\hline TLLC_LEV & -0.44 & 0.00 & 0.39 & 0.00 & 0.36 & 0.001 & -0.15 & n.s. & 0.25 & 0.02 & 0.28 & 0.01 \\
\hline
\end{tabular}

LRTA (Left/right trapezium angle); SHA (Shoulder height angle); AHA (Axilla height angle); CRIA (Clavicle-rib intersection angle); T1 tilt (T1 inclination angle); PTC (Proximal thoracic curve Cobb angle); MTC (Main thoracic curve Cobb angle); TLLC (Thoraco-lumbar/lumbar curve Cobb angle); PTC_LEV (Lower end vertebra of the proximal thoracic curve); MTC_LEV (Lower end vertebra of the major thoracic curve); TLLC_LEV (Lower end vertebra of the thoraco-lumbar/lumbar curve).

$[6,9,12]$ photography. Some authors [18] have used the linear measurement by calculating the difference in $\mathrm{cm}$ from the upper border of each acromion process to a horizontal line perpendicular to the axillary fold. This methodology requires calibration, whereby it was rejected. Furthermore, for SHA we only have reliable data from back photography [11].

2. The left/right ratio trapezium angle (LRTA) reported as the angle formed by the external border of the trapezium muscle with the horizontal. We think this could be equivalent to the Ln [L/R Trapezium Area] reported by Ono [12]. These authors found a statistically significant correlation between this parameter and the radiological variables.

Nonetheless, there are no reliable data for this measurement and, in our opinion; its calculation is excessively complex for routine use. The possibility of recording an evaluation parameter for the trapezium area was put forth by prior publications which indicate its relationship with the proximal thoracic curve.

3. Axilla height angle (AHA): formed between the line that joins the upper border of both acromion processes and the horizontal. This parameter has also been used previously $[6,11]$. It was decided to include it to analyze its possible relationship with radiological shoulder imbalance with the intention of having a second parameter to estimate shoulder imbalance for those cases when SHA is not reliable. For AHA we only have reliable data from the measurement during back photography [11].

\section{Reliability and concordance}

Most of the measurements selected revealed excellent-near perfect intra and inter observer reliability (ICC > 0.70); the inter-observer ICC were slightly less, a data already reported by other authors [19]. The reliability data are very similar for frontal and back views. Yang et al. reported somewhat more reliability (intra-observer reliability 0.97 for both measurements and inter-observer reliability 0.99 and 0.97 respectively) for the back photography [11]. The intra and inter-observer reliability values for LRTA, SHA and AHA from the front, used in our work, have not been previously published.

We found poor concordance between SHA and AHA, which suggests that one measurement cannot estimate another when analyzing the clinical balance of the shoulders. Similarly, when evaluating the concordance between front and back SHA we found poor concordance between both measurements (CCC 0.49, 95\% CI 0.32-0.64); this indicates that both measurements are not interchangeable between themselves.

\section{Relationship between the photographic and radiological measurements}

Overall, the correlations found between clinical and radiological parameters may be considered moderate to poor and in no case greater than 0.6. Behavior was similar for the three parameters evaluated (SHA, AHA and LRTA) for the two photographic views (front and back). This low correlation is similar to that reported when analyzing correlations between the radiological parameters and those obtained with the topographic analysis technique [20].

From our results, the lack of correlation between clinical $\mathrm{ShB}$ and magnitude of the PTC curve is notable; because it is usually accepted, that one of the factors that has an impact on shoulder imbalance, is the structural nature of this curve. Other previous publications had found poor or inexistent correlations between radiographic and photographic measurements in type 1 and 2 Lenke curve series $[6,9,12,21]$. These data suggest that the PTC does not have 
a significant impact on clinical shoulder balance. Conversely, we have found a moderate correlation between MTC and TLLC and the photographic measurements of the ShB, especially with the back AHA $(r=-0.44)$. Yang and Qiu found a similar correlation [6,9] and Hong et al. reported that post-operative $\mathrm{ShB}$ in a series of patients who had received surgery, was related to the correction of the MTC and TLLC [22]. These findings would indicate that clinical ShB would in part be influenced by the magnitude of the main thoracic curve and the lumbar curve. The tilt of the end vertebrae for the different curves correlated in a similar way to those overall values of the curves with the photographic measurements. No especially interesting correlation was found; therefore, the vertebra to vertebra analysis does not appear to be useful. Overall, the parameters measured in the frontal view reveal correlations with the radiographic measurements somewhat higher than those found for the rear view. Specifically, we have to point out the correlation between SHA and CRIA $(r=0.48)$ and SHA and T1-tilt $(r=0.51)$. Therefore, we would venture to recommend that the study of ShB be performed on photography taken from a frontal view, although we are aware that this shot may be a reason for conflict or rejection, especially in the case of women.

The photographic parameters (SHA, AHA, LRTA) were moderately correlated with CRIA and T1 tilt. We hypothesize that CRIA would be the radiological equivalent of SHA. Different parameters were used for the radiological measurement of ShB: Coracoids' height difference (CHD) $[5,21,23]$, clavicular angle $(\mathrm{CA})[5,23]$, clavicle-rib intersection difference (CRID) [23], radiological shoulder height (RSH) $[5,23]$ or first rib angle (FRA) $[9,12]$ among others. Our initial intention was to use clavicular angle (CA) as a radiological measure of $\mathrm{ShB}$ considering the high level of reliability of the measurement reported by Hong et al. [5]. Nonetheless, we find that for a high percentage of patients both shoulders on the $\mathrm{x}$-rays could not be observed. For this reason, we decided to use the point where the clavicle crosses the ribcage as a reference point. Bagó et al. [23] found an excellent correlation between the difference in real shoulder height and that measurement at this reference point.

The correlation between SHA and CRIA was less than expected taking into account the fact that, theoretically, both measurements evaluate the same feature. In our study, no correlations between the two parameters greater than 0.54 were found. Other authors have found similar correlations between these measures when evaluating Lenke 1 and 2 curves [9]. This low correlation cannot be attributed to the reliability of the parameters evaluated if we consider that in all works published the reliability of the photographic measurements is excellent $[6,11]$ and the same occurs with radiological measurements [5]. It is possible that the photographic measurements differ from radiological measurements because of the effect of the soft tissues in the shoulder area. It is obvious that the radiological and clinical balance of the shoulders are not an exact reflection of each other as suggested by Qiu et al. [6]; we need to evaluate both factors when analyzing shoulder balance in patients with scoliosis, not just on the Lenke 2 curves but also for all kinds of curves.

T1-tilt moderately correlates with the photographic parameters (SHA, AHA, LRTA). Therefore, shoulder position cannot be inferred from a T1 value. In fact, there is a percentage of patients in whom shoulder and $\mathrm{T} 1$ tilt are in opposite directions [24]. Other authors have found that the correlation of this measure with shoulder balance, both radiological [23] and clinical $[18,21]$ is lower than for other measures such as CA or CRID. Bearing in mind that T1 is often the upper end vertebrae of the PTC and that the magnitude of the PTC is unrelated to $\mathrm{ShB}$, our data indicate that $\mathrm{T} 1$ tilt should be the criterion to determine the structural nature of the PTC and its impact on ShB.

SHA can be considered the standard parameter to evaluate ShB in clinical photography. There is suitable intra and inter-observer reliability although the correlation with its radiographic equivalent is less than desirable. AHA is also a reliable measure but has a low correlation with radiological ShB. It is interesting to note the moderate correlation with the magnitude and tilt of the end vertebrae of the MTC which would suggest that this would be a parameter more related to deformity of the trunk than ShB. As we have pointed out above, this parameter was introduced to explore the possibility of having an alternative measure to SHA. The lack of concordance between both measures has led us to rule out this possibility. The possibility that LRTA would enable evaluating PTC led us to introduce this parameter into the analysis. In spite of correct reliability, this only shows a poor correlation with CRIA and T1 and no correlation with PTC. Although other authors have suggested that asymmetry in the trapezium area is a parameter to consider when clinically evaluating the shoulder area [12], according to our results, this is a parameter that does not provide information for SHA and AHA. Consequently, we do not believe that it makes sense to recommend use of this parameter in clinical practice.

\section{Shortcomings}

In our opinion this study presents several significant limitations. First, our study did not include analysis of the photographic parameters in relation to the scoliosis pattern. Some authors [11] have suggested that the photographic parameters could be different according to the type of curve. This possibility should be analyzed in further detail in future investigations. Second, we have not correlated $\mathrm{ShB}$ and axial plane deformity (angle of trunk inclination or apical vertebrae rotation); we take this decision due to 
the low reliability of radiographic measures used for this purpose [25]. Third, a single photograph evaluated by different observers on two occasions was used for the reliability analysis. However, the reliability of this shot was not determined. Patients were placed on floor marks and they were asked to stay in a comfortable position. We think that this methodology was sufficient to guarantee repeating the photograph. However, we cannot determine the error of measurement related to the patient's position. Fortin et al. found significant reliability of a photography technique similar to that used in our investigation $[10,19]$.

\section{Conclusions}

Clinical photography is a reliable method to evaluate clinical shoulder balance in patients with idiopathic scoliosis. Intra and inter-observer reliability is excellent; ICC greater than 0.8 were found. The reliability of the front and back views is similar although concordance analysis reveals that the measurements are not equivalent. These data confirm that $\mathrm{ShB}$ is not a pathognomonic sign of structured scoliosis. Based on the present results, the measurement of SHA does not seem an appropriate method to evaluate the effect of treatment on spinal deformity. Consequently, both examinations should be used for shoulder balance evaluation. In the future, it should be analyzed whether shoulder imbalance pattern varies according to curve pattern.

Written informed consent was obtained from the patient for the publication of this report and any accompanying images.

\begin{abstract}
Abbreviations
ShB: Shoulder balance; PTC: Cobb proximal thoracic curve; MTC: Cobb main thoracic curve; TLLC: Cobb thoracic-lumbar/lumbar curve; MLC: Major large Cobb; T1-Tilt: Inclination of T1; CRIA: Clavicle-rib intersection angle; LRTA: Left-right trapezium angle; SHA: Shoulder height angle; AHA: Armpit height angle; ICC: Intra-class correlation coefficient; SEM: Standard error of measurement; MDC: Minimal detectable change; CCC: Concordance correlation coefficient; CHD: Coracoids' height difference; CA: Clavicle angle; RSH: Radiological shoulder height; FRA: First rib angle; PTC_LEV: Proximal thoracic curve lower end vertebra; MTC_LEV: Main thoracic curve lower end vertebra; TLLC_LEV: Thoraco-lumbar/lumbar lower end vertebra.
\end{abstract}

\section{Competing interests}

The authors declare that they have no competing interests.

\section{Authors' contributions}

JB has made substantial contributions to conception and design, data measurement, data analysis and interpretation, has been involved in drafting the manuscript and revising it critically for important intellectual content, and has given final approval of the version to be published. AM has made substantial contributions to conception and design, acquisition of data, data analysis and interpretation, has been involved in the drafting of the manuscript and has given final approval of the version to be published. EA has made a substantial contribution to recruitment of patients and acquisition of data by taking the photographs and data measurements. All authors read and approved the final manuscript.

\section{Acknowledgements}

This research is supported by a research grant from Biomed/Justimplant and the Spanish Society of Spine Surgery (GEER) to perform this investigation.

\section{Author details}

'Department of Orthopaedic Surgery, Hospital Vall d'Hebron, P Vall d'Hebron, 119, 08035 Barcelona, Spain. ${ }^{2}$ Research Institute, Hospital Vall d'Hebrón, P Vall d'Hebrón, 119, 08035 Barcelona, Spain.

Received: 1 September 2014 Accepted: 30 November 2014

Published online: 14 December 2014

\section{References}

1. Negrini S, Grivas TB, Kotwicki T, Maruyama T, Rigo M, Weiss HR: Why do we treat adolescent idiopathic scoliosis? What we want to obtain and to avoid for our patients. SOSORT 2005 Consensus paper. Scoliosis 2006, 1:4.

2. Raso VJ, Lou E, Hill DL, Mahood JK, Moreau MJ, Durdle NG: Trunk distortion in adolescent idiopathic scoliosis. J Pediatr Orthop 1998, 18(2):222-226.

3. Iwahara T, Imai M, Atsuta Y: Quantification of cosmesis for patients affected by adolescent idiopathic scoliosis. Eur Spine J 1998, 7(1):12-15.

4. Theologis TN, Jefferson RJ, Simpson AH, Turner-Smith AR, Fairbank JC: Quantifying the cosmetic defect of adolescent idiopathic scoliosis. Spine 1993, 18(7):909-912.

5. Hong JY, Suh SW, Yang JH, Park SY, Han JH: Reliability analysis of shoulder balance measures: comparison of the 4 available methods. Spine 2013 , 38(26):E1684-E1690.

6. Qiu X-s, Ma W-w, Li W-g, Wang B, Yu Y, Zhu Z-z, Qian B-p, Zhu F, Sun X, Ng B-K, Cheng J-c, Qiu Y: Discrepancy between radiographic shoulder balance and cosmetic shoulder balance in adolescent idiopathic scoliosis patients with double thoracic curve. Eur Spine J 2009, 18(1):45-51.

7. Zaina F, Negrini S, Atanasio S: TRACE (Trunk Aesthetic Clinical Evaluation), a routine clinical tool to evaluate aesthetics in scoliosis patients: development from the Aesthetic Index (AI) and repeatability. Scoliosis 2009, 4:3.

8. Gorton GE 3rd, Young ML, Masso PD: Accuracy, reliability, and validity of a 3-dimensional scanner for assessing torso shape in idiopathic scoliosis. Spine 2012, 37(11):957-965.

9. Yang S, Feuchtbaum E, Werner BC, Cho W, Reddi V, Arlet V: Does anterior shoulder balance in adolescent idiopathic scoliosis correlate with posterior shoulder balance clinically and radiographically? Eur Spine J 2012, 21(10):1978-1983.

10. Fortin C, Feldman DE, Cheriet F, Gravel D, Gauthier F, Labelle H: Reliability of a quantitative clinical posture assessment tool among persons with idiopathic scoliosis. Physiotherapy 2012, 98(1):64-75.

11. Yang S, Jones-Quaidoo SM, Eager M, Griffin JW, Reddi V, Novicoff W, Shilt J, Bersusky E, Defino H, Ouellet J, Arlet V: Right adolescent idiopathic thoracic curve (Lenke $1 \mathrm{~A}$ and $\mathrm{B}$ ): does cost of instrumentation and implant density improve radiographic and cosmetic parameters? Eur Spine J 2011, 20(7):1039-1047.

12. Ono T, Bastrom TP, Newton PO: Defining 2 components of shoulder imbalance: clavicle tilt and trapezial prominence. Spine 2012, 37(24):E1511-E1516.

13. Landis JR, Koch GG: The measurement of observer agreement for categorical data. Biometrics 1977, 33(1):159-174.

14. Beaton DE: Understanding the relevance of measured change through studies of responsiveness. Spine 2000, 25(24):3192-3199.

15. Lin L-I-K: A concordance correlation coefficient to evaluate reproducibility. Biometrics 1989, 45:255-268.

16. Mc Bride G: A Proposal for Strength-of-Agreement Criteria for Lin's Concordance Correlation Coefficient. In NIWA Client Report: HAM2005-062; 2005. http:www.niwa.co.nz.

17. Lenke LG, Betz RR, Harms J, Bridwell KH, Clements DH, Lowe TG, Blanke K: Adolescent idiopathic scoliosis: a new classification to determine extent of spinal arthrodesis. JBJS Am 2001, 83-A(8):1169-1181.

18. Akel I, Pekmezci M, Hayran M, Genc Y, Kocak O, Derman O, Erdogan I, Yazici M: Evaluation of shoulder balance in the normal adolescent population and its correlation with radiological parameters. Eur Spine J 2008, 17(3):348-354.

19. Fortin C, Ehrmann Feldman D, Cheriet $F$, Labelle $\mathrm{H}$. Clinical methods for quantifying body segment posture: a literature review. Disabil Rehabil 2011, 33(5):367-383.

20. Patias P, Grivas TB, Kaspiris A, Aggouris C, Drakoutos E: A review of the trunk surface metrics used as Scoliosis and other deformities evaluation indices. Scoliosis 2010, 5:12 
21. Kuklo TR, Lenke LG, Graham EJ, Won DS, Sweet FA, Blanke KM, Bridwell KH: Correlation of radiographic, clinical, and patient assessment of shoulder balance following fusion versus nonfusion of the proximal thoracic curve in adolescent idiopathic scoliosis. Spine 2002, 27(18):2013-2020.

22. Hong JY, Suh SW, Modi HN, Yang JH, Park SY: Analysis of factors that affect shoulder balance after correction surgery in scoliosis: a global analysis of all the curvature types. Eur Spine J 2013, 22(6):1273-1285.

23. Bagó J, Carrera L, March B, Villanueva C: Four radiological measures to estimate shoulder balance in scoliosis. J Pediatr Orthop B 1996, 5(1):31-34.

24. Itharreborde B, Even J, Lefevre Y, Fitoussi F, Presedo A, Souchet P, Penneçot GF, Mazda K: How to determine the upper level of instrumentation in Lenke types 1 and 2 adolescent idiopathic scoliosis: a prospective study of 132 patients. J Pediatr Orthop 2008, 28(7):733-739.

25. Morrison DG, Chan A, Hill D, Parent EC, Lou EH: Correlation between Cobb angle, spinous process angle (SPA) and apical vertebrae rotation (AVR) on posteroanterior radiographs in adolescent idiopathic scoliosis (AIS). Eur Spine J, in press.

doi:10.1186/s13013-014-0023-6

Cite this article as: Matamalas et al: Reliability and validity study of measurements on digital photography to evaluate shoulder balance in idiopathic scoliosis. Scoliosis 2014 9:23.

\section{Submit your next manuscript to BioMed Central and take full advantage of:}

- Convenient online submission

- Thorough peer review

- No space constraints or color figure charges

- Immediate publication on acceptance

- Inclusion in PubMed, CAS, Scopus and Google Scholar

- Research which is freely available for redistribution 